\title{
An automated positive reward method for measuring acoustic sensitivity in fish
}

\author{
HONG Y. YAN and ARTHUR N. POPPER \\ University of Maryland, College Park, Maryland
}

\begin{abstract}
A microcomputer-controlled positive reward operant conditioning paradigm was developed to measure auditory sensitivity of the goldfish (Carassius auratus). Although other procedures have been used to measure hearing sensitivity in fish, all earlier studies have been done with some form of aversive conditioning. Goldfish were trained with a positive reinforcement method to determine hearing thresholds at $200,500,1000,1500$, and $2000 \mathrm{~Hz}$. The audiogram obtained in this study is in close agreement with thresholds obtained with instrumental avoidance and classical conditioning procedures involving negative reinforcement (shock). The similarity in threshold data validates the effectiveness of the positive reinforcement paradigm described here. The major advantages of the present paradigm are the following: (1) No physical punishment such as electric shock is used, and (2) the versatility of the system allows easy modification for a variety of experiments.
\end{abstract}

The hearing capabilities of fish have been investigated since the early 1900s (Parker, 1902, 1903). Quantitative work on the range of frequencies over which fish can hear was first carried out by von Frisch and his colleagues (von Frisch, 1936). This laid the foundation for more comprehensive psychophysical analyses (e.g., threshold determinations, acoustic intensity limens) of fish hearing (Jacobs \& Tavolga, 1967; Tavolga \& Wodinsky, 1963; see also comments in Tavolga, 1976).

The measure of hearing sensitivity is generally expressed in terms of the minimum detectable level, or threshold. This is a statistical value that is defined as the sound pressure level (SPL) at which the animal responds correctly in a specified proportion of presentations (e.g., $50 \%$ ). A plot of thresholds for a range of frequencies is called an audiogram (Hawkins, 1981). Audiograms are currently available for about 52 fish species, including goldfish, carps, cods, catfish, damselfishes, minnows, knifefish, and sharks (see Fay, 1988, for a detailed list).

Behavioral audiograms of fish have generally been obtained with one of three different procedures. The simplest method involved training fish to approach a particular area on hearing a sound. A correct response was rewarded with food (Kritzler \& Wood, 1961). Because of the random location of the fish relative to the sound

We are grateful to R. J. Dooling for invaluable suggestions and dis cussions that led to the development of this procedure. E. E. Allen kindly shared his idea about system construction. F. Hanson and H. C. Chen provided assistance in input/output board programming. E. Clark, C. Platt, J. Presson, C. Hue, P. Edds, and three anonymous reviewers offered valuable comments on the manuscript. This work was supported by grants from the National Institutes of Health (DC-00140) and the Office of Naval Research (N-00014-87-K-0604). Reprint requests should be sent to Hong $Y$. Yan, Department of Zoology, University of Maryland, College Park, MD 20742. Copies of the software may also be obtained by request. source when the sound was presented, it was difficult to determine the SPL detected by the fish. In addition, the response of the fish was often ambiguous and erratic, especially near the threshold for the frequency being studied (Hawkins, 1981).

The second method consisted of instrumental-that is, shuttlebox (Horner, Longo, \& Bitterman, 1961)avoidance conditioning, in which fish were trained to cross a barrier in the tank on hearing a sound. The fish thereby avoided getting an electric shock (Tavolga \& Wodinsky, 1963). This method also suffered from some nonuniformity of the sound field, because of the free movement of the fish around the tank.

The third and most widely used method was the classical conditioning of heartbeat or gill ventilation (Fay, 1969), in which the sound stimulus was paired with an electric shock. The fish were conditioned to associate the sound with the shock, which resulted in cardiac and ventilatory suppression whenever the stimulus was detected. In such studies, fish could be positioned at a specific point in the sound field where the sound level was known. Although this method gave repeatable data, the procedure was aversive not only because a shock was used to condition the fish, but also because the animal had to be restrained. The restraining of fish tends to lead to physiological stress (Donaldson, 1981). In addition, not every fish species could be conditioned with electric shockfor example, a shark (Nelson, 1967), or a cichlid fish (Allen \& Fernald, 1985).

Operant conditioning procedures involving positive reinforcement have proved enormously successful in the investigation of sensory processes in laboratory animals such as goldfish (for color vision, see Yager \& Thorpe, 1970), cichlid fish (for spectral sensitivity, Allen \& Fernald, 1985), rats, pigeons, cats, and monkeys (see the review in Stebbins, 1970). However, no such procedures 
have been applied to the study of acoustic sensitivity in fish. Our purpose in the present study was to develop an automated operant conditioning protocol in which positive reinforcement was used to measure fish acoustic sensitivity, without recourse to an aversive method.

\section{METHOD}

\section{Subjects}

Five goldfish (Carassius auratus), about $8 \mathrm{~cm}$ in standard length (measured from tip the of snout to the end of the vertebral column) were obtained from a local aquarium supplier. Each fish was housed in an individual 40-1 glass aquarium $(50 \times 30 \times 26.6 \mathrm{~cm})$. The water was continuously aerated and filtered.

\section{Apparatus}

The test apparatus sat on a Plexiglas platform $(32 \times$ $20 \mathrm{~cm}$ ) attached to a support made from wooden studs, which provided a structure that could be transferred easily from one tank to another (Figure 1). An automatic feeder (solenoid-controlled) on the platform dispensed pellets of fish food on command from a one-shot timer (Coulbourn Instruments, S52-53, Lehigh Valley, PA) controlled by the computer. These floating food pellets (Hikari Gold, Kyorin Food, Himeji, Japan) were dispensed through a plastic tube that contacted the surface of the water, so that a fixed feeding station (Figure 1, interface of $T$ and water surface) was easily recognized by the fish. A $10-\mathrm{W}$ ceiling light (C-light) was hung at the rear of the platform to illuminate the tank during the experiment when needed.

The response panel consisted of two steel rods $6 \mathrm{~cm}$ apart. These were attached to the underside of the platform. Each rod held a sealed clear plastic tube (diameter $1 \mathrm{~cm}$, length $5 \mathrm{~cm}$ ) that contained a miniature $10-\mathrm{W}$ light

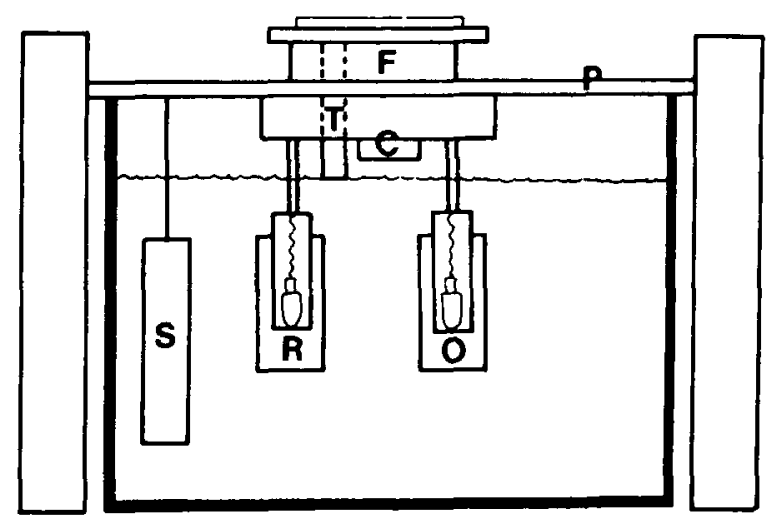

Figure 1. Schematic diagram of the test tank from fish-eye view, showing the apparatus for measuring hearing thresholds. $\mathrm{C}=$ ceiling light; $F=$ automatic feeder; $O=$ observation paddle; $P=$ Plexiglas platform; $R=$ report paddle; $S=$ underwater speaker; $T=$ pellet delivery tube. Notice that both the $\mathbf{O}$-light and the $\mathbf{R}$-light are housed inside clear plastic waterproof tubes. See text for a detailed description of the apparatus. bulb. A piece of pressure-sensitive Kynar piezo film (Pennwalt Co., Valley Forge, PA) was attached outside each tube to detect the responses of the fish. One tube with piezo film was the observation paddle (O-paddle) and the other the report paddle (R-paddle). The piezo film only required a gentle touch ( $<2 \mathrm{~g}$ of force) of the paddle by the fish in order to trigger an analog response signal to an analog/digital converter (Dual threshold detector, $\mathrm{CI}$ S21-10) (Figure 2).

Sounds were generated with a signal generator (CI S8106) connected to a digitally controlled attenuator (CI S8508) and an audio mixer-amplifier (CI S82-24). The stimuli were presented through an underwater speaker (University Sound UW-30, Oklahoma City, OK), which was attached by wires to the underside of the Plexiglas platform. The signal generator and attenuator were calibrated with an LC-10 hydrophone (Celesco, Canoga Park, CA) and a wave analyzer (Hewlett-Packard 3581 A; see Figure 2). SPLs of various frequencies and attenuations were also measured and calibrated around the $\mathrm{O}$-paddle. These data served as a reference when specific frequency and SPLs were used later in the acoustic sensitivity study.

A 24-bit parallel digital input/output board (Metrabyte PIO12, Taunton, MA) was used as an interface between a microcomputer (IBM AT) and all electronic modules. The apparatus, training of animals, and acoustic sensitivity study were all controlled with programs written in Microsoft QuickBASIC (Version 4.5).

Experiments were conducted in a soundproof chamber $(2.1 \times 2 \times 2.2 \mathrm{~m})$ (Industrial Acoustic Corp., Bronx, NY) to cut down on background noise and to prevent masking of absolute thresholds. Measurement of the SPL inside the chamber showed that the background noise was well below any threshold value obtained in the present experiment.

\section{Training Procedure}

Autoshaping procedures were used to train the goldfish to make the appropriate series of responses. The training procedure was divided into five stages. In Stage 1, food pellets were dropped into the feeding station (Figure 1) at random intervals, so that the fish would recognize the location of the feeding site. In Stage 2, a few food pellets were placed inside the clear plastic tube of the O-paddle. The fish were deprived of food for $23 \mathrm{~h}$ to motivate them to peck the O-paddle. Pecking of the $\mathrm{O}$ - paddle triggered the automatic feeder, and the fish thus learned to associate hitting the $\mathrm{O}$-paddle with getting fed.

In Stage 3, no food pellets were inside the clear tube attached to the O-paddle. Instead, the O-paddle light was first turned on. After the fish pecked at the O-paddle, the light was turned off and the R-paddle light was turned on. If the R-paddle was pecked within a certain time limit ( $5 \mathrm{~min}$ in the beginning, which was then gradually reduced to $30 \mathrm{sec}$ ), the R-paddle light was turned off and the fish was rewarded with food. If the R-paddle was not pecked within the time limits or the R-paddle was pecked before the $\mathrm{O}$-paddle, the ceiling light was turned off and the fish 


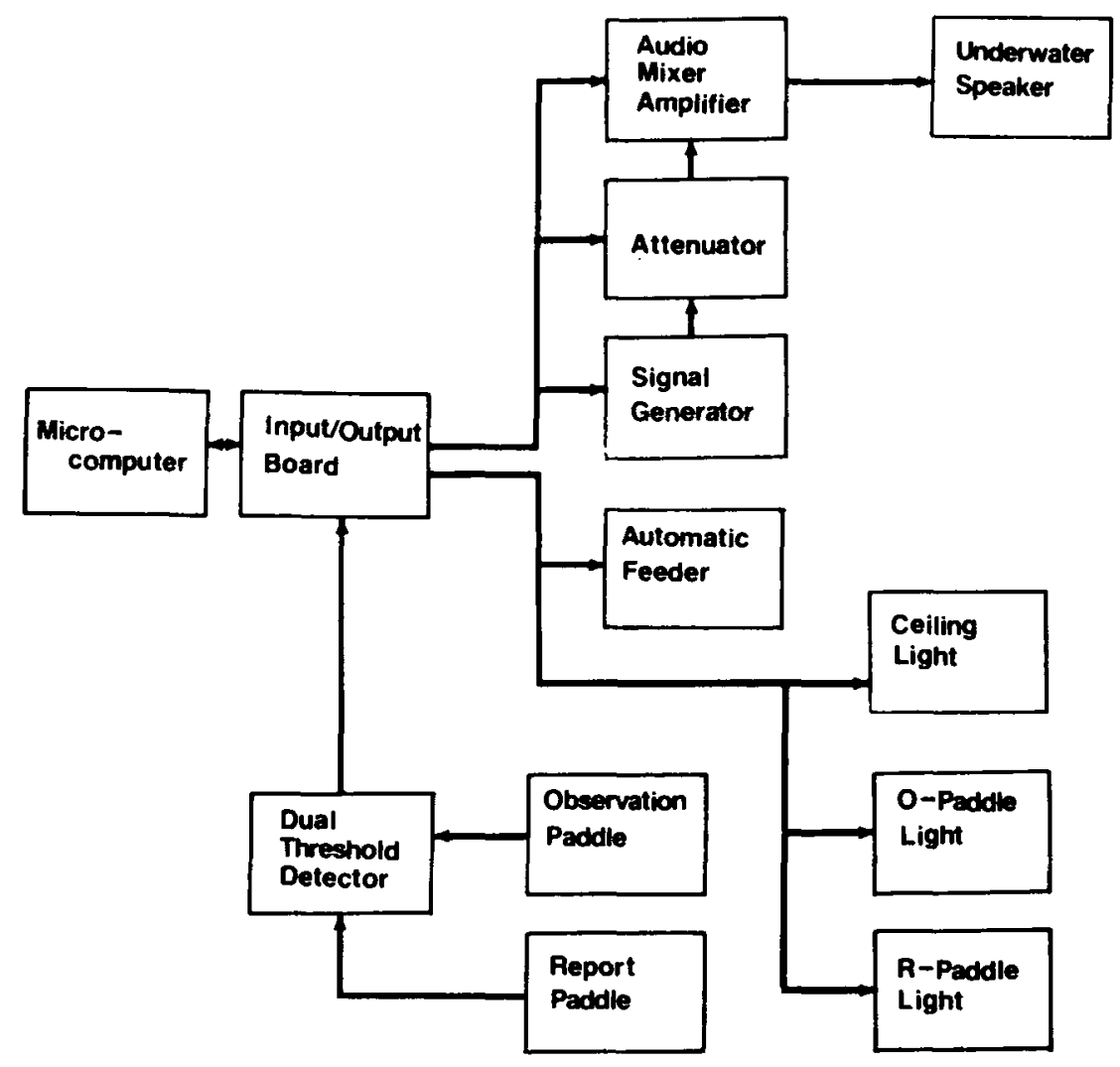

Figure 2. Block diagram of the automated electronic system and apparatus for behavioral acoustic sensitivity study.

were subjected to complete darkness for $90 \mathrm{sec}$; this was termed a blackout. The complete darkness deprived the fish of feeding opportunities and functioned to deter the fish from making mistakes.

Stage 4 of training was carried out with an almost identical protocol to that for Stage 3, except that the pecking of the O-paddle was followed by a $500-\mathrm{Hz}$ tone $(13 \mathrm{~dB}$, re: $1 \mu \mathrm{bar}$ ) instead of the lighting of the R-paddle. The fish had to peck the R-paddle when the sound was on. At the beginning, the sound duration was up to $90 \mathrm{sec}$ long, and then it was gradually reduced to $4 \mathrm{sec}$.

At the final stage of training (Stage 5), blank trials (i.e., a period of silence) were presented in random order and in equal numbers with sound presentations. The pecking of the R-paddle during blank trial, also led to a blackout. Therefore, the purpose of the blank trial was to train the fish not to peck the R-paddle when sound was not heardthat is, when it was below threshold. Training continued until the fish constantly attained a combination level of $90 \%$ correct response and a false alarm rate of less than $10 \%$ over a session of 32 trials. The false alarm rate was calculated by dividing the number of responses to the report paddle when no tone was presented by the total number of observing responses (Okanoya \& Dooling, 1987).

\section{Acoustic Sensitivity Study}

Fish were deprived of food for 16-23 h before a test. The test apparatus was placed onto the home tank for each fish at the time of the test. The tank and apparatus were placed in the soundproof chamber during threshold determinations. A total of 5 goldfish were used during the experiments. However, due to disease, only 2 were able to go through all acoustic sensitivity measurements and hence only data from these 2 fish were used for analysis.

Thresholds were measured at 200,500,1000,1500 and $2000 \mathrm{~Hz}$, using a modified method of constant stimuli (e.g., Yost \& Nielsen, 1985). Six SPLs were used for testing at each frequency. The SPLs were selected on the basis of preliminary experiments that gave a general sense of the threshold. The experimental SPLs bracketed the preliminary threshold and were modified if the actual results indicated that the threshold levels were not being appropriately bracketed. In each test run, five replicates of a chosen SPL and five blank trials were randomly presented to the fish. If there were more than three false responses (i.e., the fish pecked the R-paddle when the blank was on) in a single test run, the data for that particular run was discarded and the run was repeated later. At each intensity, a minimum of three test runs were repeated for each fish in order to calculate the response 


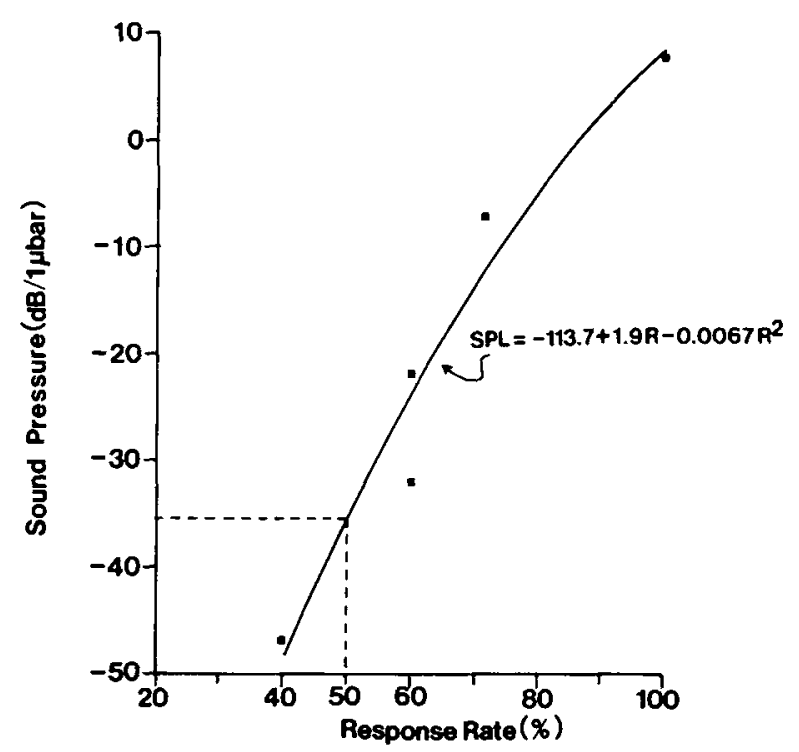

Figure 3. A typical example showing threshold determination at $1000 \mathrm{~Hz}$ for Goldfish 1 . A best-fit polynomial function, SPL = $-113.7+1.90 R-0.0067 R^{2}$, is generated on the basis of response rate ( $R$, in percent) and sound pressure level (SPL, in decibels). The threshold for this particular frequency is calculated by noting the intensity that yields a $50 \%$ ( $R$ value) proportion of responses from the equation-that is, $-35.5 \mathrm{~dB}$. rate $(R)$. Response rate was calculated by dividing the number of correct responses to the report paddle by the total number of trials presented.

On the basis of the $R$ of each SPL tested, a polynomial psychometric function was generated in the form of SPL $(\mathrm{dB})=a+b R(\%)+c R^{2}(\%)$ to relate the proportion of the $R$ value (in percent) to the stimulus values (SPL) (in decibels) (Richards, 1976). A polynomial function was chosen because of a better fit-that is, a higher correlation coefficient than linear function for the data set. The threshold for a particular frequency was obtained by calculating the SPL that yielded a 50\% proportion of responses in the psychometric function (see Figure 3 for an example).

\section{RESULTS}

The threshold SPLs for five frequencies $(200,500$, 1000,1500 , and $2000 \mathrm{~Hz}$ ) obtained from the goldfish with the modified constant stimuli method are shown in Figure 4. The lowest mean threshold was $-51 \mathrm{~dB}$ ( $S D$ $= \pm 13.6)$ at $500 \mathrm{~Hz}$. Threshold SPLs for 200 and $1000 \mathrm{~Hz}$ were $-32 \mathrm{~dB}(S D= \pm 9.8)$, and $-35 \mathrm{~dB}(S D$ $= \pm 2.5)$, respectively. However, an increase of SPL at $1500 \mathrm{~Hz}(-29 \mathrm{~dB} ; S D= \pm 7.0)$ was found. At $2000 \mathrm{~Hz}$, mean threshold rose to $4 \mathrm{~dB}(S D= \pm 4.0)$. The

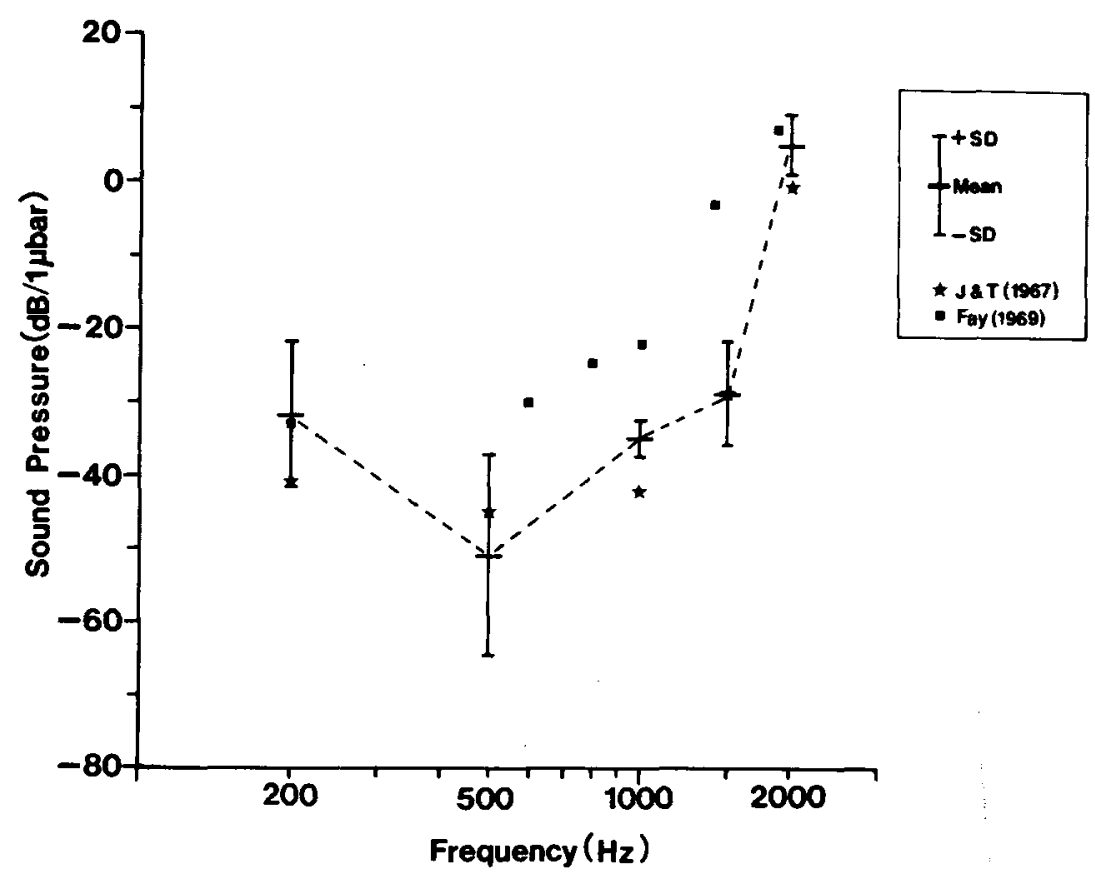

Figure 4. Behavioral audiogram for goldfish, measured by positive reinforcement method. Each mean (and standard deviation) threshold value in the present study represents an average of six measurements (three from each fish). Asterisks indicate mean threshold values obtained by Jacobs and Tavolga (1967) with an instrumental avoidance procedure. Solid blocks indicate mean threshold values obtained by Fay (1969) with classical conditioning of respiration suppression. 
overall results indicated that the most sensitive range of pure tone hearing of goldfish was between 200 and $1000 \mathrm{~Hz}$.

\section{DISCUSSION}

Our purpose in the present study was to develop an operant conditioning paradigm that would provide a reliable measure of the hearing capabilities of fish, without subjecting the fish to aversive shock conditioning. In order to ensure that our procedure gives reliable data, we have compared our results with the most widely cited data, which were obtained by Jacobs and Tavolga (1967) with an instrumental avoidance procedure, and to a classical conditioning procedure that was used by Fay (1969) (see Figure 4). Our data, with lower thresholds, appear to be more sensitive than Fay's. This difference may have been caused by the stress from restraining the fish in cardiac/gill conditioning. However, the audiogram of goldfish obtained here follows the general trend of audiograms of goldfish previously reported by several different workers who used either avoidance conditioning or classical conditioning methods (see the review by Popper \& Fay, 1973; see also Fay, 1988). As indicated by Popper, Chan, and Clarke (1973), direct comparison of threshold data may not be appropriate, owing to the various acoustic conditions and different sizes of fish. However, despite completely different paradigms (positive reinforcement vs. classical conditioning), the overall results agree with the published data, and this certainly validates the effectiveness of the positive reinforcement paradigm employed in the present study.

Nonuniformity of the sound field encountered in the instrumental avoidance conditioning method is avoided in the present paradigm, for the fish always heard the sound at the same spot (O-paddle) for sound presentation. This spot could then be thoroughly calibrated. In addition, the fish were allowed to swim freely in the tank, preventing physiological stress from restraint in the classical conditioning of cardiac and/or ventilatory suppression.

Classical conditioning of heartbeat or gill ventilation is often complete within 10 trials; it can be achieved within $1 \mathrm{~h}$ after the start of training. Thus, several thresholds can be determined in a day (Hawkins, 1981). With the present reward reinforcement training method, however, it takes about 50 trials (a correct response to either the $\mathrm{O}$-paddle or the R-paddle is a trial) to train a fish. On the average, 3-5 days are required to train a fish. However, individual differences, ranging from 2 to 7 days, in the time to complete the training process have been observed. The complexity of the paradigm, which involves two separate responses ( $\mathrm{O}$-paddle and $\mathrm{R}$-paddle pecking) and control by the fish of the intertrial period in the beginning of training session, prolongs the training period. Our experience indicates that the whole training process could be shortened by decreasing the intertrial interval. In the preliminary experiments, it was observed that a long (i.e., $90 \mathrm{sec}$ ) blackout period had a significantly lower false alarm rate $(<10 \%)$ than that $(>40 \%)$ of a shorter $(30-\mathrm{sec})$ blackout period. Once a goldfish is well trained, one or two thresholds can be determined in a single day.

Food-reward reinforcement has proved effective in mammalian auditory studies but has not enjoyed a similar success with fishes. Stebbins (1983) attributed this disparity in fish to the difficulty in maintaining motivation over a lengthy test session and to the food delivery problems underwater. In the present study, each food pellet weighed only about $2 \mathrm{mg}$, and an $8-\mathrm{cm}$ goldfish (body weight $20 \mathrm{~g}$ ) could take up to 150 pellets a day and still maintain high motivation. In addition, floating food pellets could be confined to a fixed feeding station, thereby solving the underwater food delivery problem.

A light cue (such as the $\mathrm{O}$-paddle or $\mathrm{R}$-paddle light) is used in the present experiment to facilitate training process. However, the light cue may not be equally effective for nocturnal species such as catfish. Our preliminary data indicate that gluing food pellets to the surface of the O-paddle can enable the best use of the chemosensory ability of fish to peck at the O-paddle without a light cue to establish the conditioning response.

The most sensitive microswitch on the market (Microswitch Inc., Serial No. 311SM703-T) requires at least $6 \mathrm{~g}$ of force for a response to be registered (Park, Okanoya, \& Dooling, 1985). Our preliminary tests show that only goldfish longer than $15 \mathrm{~cm}$ (standard length) can exert enough force to trigger the microswitch. In contrast, the piezo film requires less than $\mathbf{2} \mathrm{g}$ of force (Kynar piezo film, Product Data No. 9, Pennwalt Co.), so it is easy for a small goldfish (less than $5 \mathrm{~cm}$ in standard length) to register a response. Through the coupling of the piezo film to a Coulbourn dual threshold detector (CI S21-10), the sensitivity of the film can be adjusted to meet different pecking forces from different sizes of fish. In addition, piezo films are inexpensive $(\$ 3.00$ each) and commercially available, and they can be plugged into any analog/digital converter without the elaborate wiring that is required by phonograph levers (Longo \& Bitterman, 1959) or strain gauges (Couvillon \& Bitterman, 1985).

The present automated reward system offers several advantages over the instrumental avoidance and classical conditioning method. (1) The positive reinforcement operant conditioning imposes no physical punishment (such as electric shock) to fish, thereby permitting a more humane experiment. In addition, no restraint of the fish in a fixed position is needed. (2) The operating system is automated with the aid of a microcomputer, and the programming can be done with any programming language that is supported by the input/output board. (3) Because the fish are trained to come to the same spot for sound presentations, the problem of nonuniformity of the sound field is avoided. (4) An inexpensive input/output board allows up to 24 channels of bidirectional control of electronic models, which adds to the versatility of the system and allows easy modification for a variety of experimental needs. 
The similarity between our data and those obtained by other workers validates the new procedure. Most important, it is now possible to use this procedure for measures of other aspects of fish hearing, such as masking and discrimination ability.

\section{REFERENCES}

Allen, E. E., \& Fernald, R. D. (1985). Spectral sensitivity of the African cichlid fish, Haplochromis burtoni. Joumal of Comparative Physiology A, 157, 247-253.

Couvillon, P. A., \& Bitterman, M. E. (1985). Effect of experience with a preferred food on consummatory responding for a less preferred food in goldfish. Animal Learning \& Behavior, 13, 433-438.

Donaldson, E. M. (1981). The pituitary-interrenal axis as an indicator of stress in fish. In A. D. Pickering (Ed.), Stress and fish (pp. 1147). London: Academic Press.

FAY, R. R. (1969). Behavioral audiogram for the goldfish. Journal of Auditory Research, 9, 112-121.

FAY, R. R. (1988). Hearing in vertebrates: A psychophysics databook. Winnetka, IL: Hill-Fay Associates.

HawkIns, A. D. (1981). The hearing abilities of fish. In W. N. Tavolga, A. N. Popper, \& R. R. Fay (Eds.), Hearing and sound communication in fishes (pp. 109-137). New York: Springer.

Horner, J. L., Longo, N., Bitrerman, M. E. (1961). A shuttlebox for fish and a control circuit of general applicability. American Journal of Psychology, 74, 114-120.

JACOBS, D. W., TAvolga, W. N. (1967). Acoustic intensity limens in the goldfish. Animal Behaviour, 15, 324-335.

Kritzler, H., \& WoOd, L. (1961). Provisional audiogram for the shark, Carcharhinus leucas. Science, 133, 1480-1482.

LoNGo, N., \& BitTERMAN, M. E. (1959). Improved apparatus for the study of learning in fish. American Joumal of Psychology, 72, 612-620.

Newson, D. R. (1967). Cardiac responses to sounds in the lemon shark, Negaprion brevirostris. In P. W. Gilbert, R. F. Mathewson, \& D. P. Rall (Eds.), Sharks, skates, and rays (pp. 533-544). Baltimore: Johns Hopkins University Press.

OKANOYA, K., \& DoOLING, R. J. (1987). Hearing in passerine and psit- tacine birds: A comparative study of absolute and masked auditory thresholds. Journal of Comparative Psychology, 101, 7-15.

Park, T., Okanoya, K., \& Dooling, R. J. (1985). Operant conditioning of small birds for acoustic discrimination. Joumal of Ethology, 3, 5-9.

PARKER, G. H. (1902). Hearing and allied senses in fishes. Bulletin of the United States Fishery Commission, 22, 45-64.

PARKer, G. H. (1903). The sense of hearing in fishes. American Naturalist, 37, 185-204.

Popper, A. N., Chan, A. T. H., Clarke, N. L. (1973). An evaluation of methods for behavioral investigations of teleost audition. Behavior Research Methods \& Instrumentation, 5, 470-472.

Popper, A. N., \& FAY, R. R. (1973). Sound detection and processing by teleost fishes: A critical review. Journal of the Acoustical Society of America, 53, 1515-1529.

RICHARDS, A. M. (1976). Basic experimentation in psychoacoustics. Baltimore: University Park Press.

Stebrins, W. C. (1970). Animal psychophysics: The design and conduct of sensory experiments. New York: Appleton-Century-Crofts.

Stebrins, W. C. (1983). The acoustic sense of animals. Cambridge, MA: Harvard University Press.

TAvoLGA, W. N. (1976). Sound perception in fishes: Benchmark papers in animal behavior (Vol. 7). Stroudsburg, PA: Dowden, Hutchinson \& Ross.

Ta volgA, W. N., \& Wodinsky, J. (1963). Auditory capacities in fishes. Pure tone thresholds in nine species of marine teleosts. Bulletin of the American Museum of Natural History, 126, 177-240.

von Frisch, K. (1936). Über den Gehörsinn der Fische. Biological Review, 11, 210-246.

YAGER, D., THORPE, S. (1970). Investigations of goldfish color vision. In W. C. Stebbins (Ed.), Animal psychophysics: The design and conduct of sensory experiments (pp. 259-275). New York: AppletonCentury-Crofts.

Yost, W. A., \& NielseN, D. W. (1985). Psychophysics: Discrimination procedures. In W. A. Yost \& D. W. Nielsen (Eds.), Fundamentals of hearing (2nd ed., pp. 109-122). New York: Holt, Reinhart \& Winston.

(Manuscript received December 17, 1990; revision accepted for publication March 25, 1991.) 